\title{
Prevention of treatment-induced ototoxicity: An update for clinicians
}

\author{
L Ramma, ${ }^{1}$ AUD, MPH; N Schellack, ${ }^{2}$ BCur, BPharm, PhD; B Heinze, ${ }^{3}$ M(ECI), PhD \\ ${ }^{1}$ Division of Communication Sciences and Disorders, Department of Health and Rehabilitation Sciences, Faculty of Health Sciences, \\ University of Cape Town, South Africa \\ ${ }^{2}$ Department of Clinical Pharmacy, School of Pharmacy, Sefako Makgatho Health Sciences University, Pretoria, South Africa \\ ${ }^{3}$ Department of Speech-Language Pathology and Audiology, Faculty of Humanities, University of Pretoria, South Africa
}

Corresponding author: L Ramma (lebogang.ramma@uct.ac.za)

\begin{abstract}
Ototoxicity is a common side-effect during treatment of a a number of life-threatening health conditions. Often, when treating such conditions, the focus tends to be on saving the patient's life rather than preserving their hearing or vestibular function. However, advances in clinical management of these conditions, availability of less ototoxic alternative medicines and developments in interventions to prevent ototoxicity now give clinicians a better opportunity to save the patient's life and preserve their hearing and vestibular function. Effective communication between audiologists, clinical pharmacists and physicians is critical in preventing drug-induced ototoxicity. Clinicians need to stay up to date regarding scientific and clinical developments related to the prevention of treatment-induced ototoxicity. This article reviews common ototoxic medicinal agents and strategies to prevent ototoxicity, and discusses recent developments towards preventing drug-induced ototoxicity.
\end{abstract}

S Afr Med J 2019;109(3):145-149. DOI:10.7196/SAMJ.2019.v109i3.13903

Unwanted adverse effects from drugs as part of medical treatment are common. ${ }^{[1]}$ It is therefore as important for clinicians to recognise adverse drug reactions (ADRs) as it is to diagnose serious diseases if effective clinical management is to be realised. ${ }^{[2]}$ Ototoxicity is an adverse reaction resulting from the pharmacological treatment of many life-threatening illnesses, such as cancer, multidrug-resistant tuberculosis and congestive heart failure, ${ }^{[2]}$ and it is increasingly becoming a problem in low- and middle-income countries (LMICs) such as South Africa (SA). Some ototoxic medicines primarily affect the auditory system (cochleotoxic), while others primarily affect the vestibular system (vestibulotoxic), and some classes, e.g. aminoglycosides, affect both systems. ${ }^{[3]}$

The reported incidence of ototoxic side-effects varies extensively depending on several variables: drug type, classification criteria for ototoxicity and patients' demographic profiles. The incidence ranges from $18 \%$ (for some aminoglycosides) to $100 \%$ (for some platinum compounds, e.g. cisplatin). ${ }^{[4,5]}$ There is considerable variation in individual susceptibility to drug-induced ototoxicity. Table 1 provides a list of some of the risk factors associated with ototoxicity and primarily focuses on commonly prescribed drugs, such as the aminoglycosides, chemotherapeutic agents and loop diuretics. ${ }^{[6,7]}$

The onset of ototoxicity symptoms varies from a few days to months after systemic administration. ${ }^{[8]}$ Ototoxicity may present as cochleotoxicity or vestibulotoxicity or both. Cochleotoxicity may exhibit hearing loss, which could be permanent, tinnitus or hyperacusis (increased sensitivity to everyday sounds), as well as difficulty with speech discrimination, especially in the presence of background noise. Vestibulotoxicity may present as general disequilibrium, unsteadiness when walking or ataxic gait, oscillopsia and nystagmus. ${ }^{[8,9]}$

Iatrogenic hearing loss resulting from ototoxicity can have a negative impact on affected individuals. Patients may experience communication difficulties, which could lead to isolation, depression and diminishing integration in a wider society. ${ }^{[10]}$ Likewise, loss of vestibular function due to ototoxicity can impair one's ability to perform activities of daily living and integration within society, thus resulting in depression, cognitive decline and diminished quality of life. ${ }^{[11]}$

Treatment regimens that include ototoxic pharmacological agents require clinicians to navigate the delicate balance of remedying the primary health condition and ensuring that the patient's hearing and vestibular status are not negatively affected. Fortunately, improvements in pharmacological management of patients who are treated with such regimens now present clinicians with more options to prevent ototoxicity. Therefore, patients should not be burdened with the task of making the unenviable choice of 'death or deaf'. Physicians should explore all the available options for preventing treatment-induced ototoxicity.

\section{Ototoxic medicines}

Many medicines are known to have some degree of ototoxic effect. While it is impossible for a clinician to remember all of these, certain drug classes such as the aminoglycosides and some platinum compounds are known to be associated with a higher incidence of ototoxicity - often with serious and irreversible consequences. Most recently, the newer generation of medicines, such as cyclodextrins (e.g. 2-hydroxypropyl- $\beta$-cyclodextrin $(\mathrm{HP} \beta \mathrm{CD})$ ), have also been reported to be associated with some ototoxic effects. ${ }^{[12]}$ Table 2 presents an overview of some medicines with known ototoxic sideeffects. It is not an exhaustive list, but can serve as a quick reference.

\section{Prevention of pharmacotherapy- induced ototoxicity}

There is currently no phenotypic marker that can be used by clinicians to reliably predict the likelihood of developing ototoxicity after treatment. There is a paucity of evidence of the relationship 
Table 1. Risk factors and symptoms associated with ototoxicity

\begin{tabular}{|c|c|c|}
\hline Drug category & Risk factors & Symptoms \\
\hline Aminoglycosides & $\begin{array}{l}\text { Therapy lasting }>7 \text { days } \\
\text { Prior exposure to aminoglycosides } \\
\text { High daily doses } \\
\text { Age extremes ( }<5 \text { years and }>60 \text { years) } \\
\text { Presence of specific mitochondrial mutations } \\
\text { Exposure to loud sounds } \\
\text { Dehydration } \\
\text { Prematurity }\end{array}$ & $\begin{array}{l}\text { Permanent high-frequency sensorineural } \\
\text { hearing loss } \\
\text { Oscillopsia and chronic dizziness/disequilibrium }\end{array}$ \\
\hline Platinum compounds (i.e. cisplatin) & $\begin{array}{l}\text { Age extremes } \\
\text { Pre-existing hearing loss } \\
\text { Dose, duration and route of administration } \\
\text { Cranial irradiation } \\
\text { Concomitant use of other ototoxic agents } \\
\text { Dehydration }\end{array}$ & $\begin{array}{l}\text { Permanent high-frequency sensorineural } \\
\text { hearing loss } \\
\text { High-pitched tinnitus }\end{array}$ \\
\hline Loop diuretics & $\begin{array}{l}\text { Renal impairment } \\
\text { Prematurity } \\
\text { Concomitant use of aminoglycoside antibiotic } \\
\text { Dehydration }\end{array}$ & $\begin{array}{l}\text { Sudden progressive sensorineural } \\
\text { hearing loss (flat configuration) - may be } \\
\text { transient or permanent } \\
\text { Oscillopsia and chronic dizziness/disequilibrium }\end{array}$ \\
\hline
\end{tabular}

between ototoxicity, drug dosage, peak serum levels and renal toxicity. ${ }^{[14]}$ Therefore, the only way to prevent pharmacotherapy-induced ototoxicity is to monitor patients during treatment. Cochleotoxicity monitoring involves prospective collection of serial audiometric data at regular intervals to ensure early detection of changes in hearing thresholds presumably attributed to the treatment regimen. Ototoxic damage typically starts in the cochlear base and progresses towards the apex. Hence, cochleotoxicity-monitoring protocols must include audiological tests that are sensitive to changes in ultra-high-frequency thresholds (i.e. test frequencies $>8 \mathrm{kHz}$ ) and/or otoacoustic emissions. ${ }^{[15]}$ It is important that baseline audiometric assessment is completed before administration of ototoxic medications. If this is not possible, baseline assessment should occur within 24 hours of cisplatin administration and within 72 hours of the initial treatment dose for aminoglycosides. ${ }^{[15]}$ Symptoms of vestibulotoxicity (oscillopsia and disequilibrium) may be less obvious than the perception of tinnitus and hearing difficulties, especially if the patient is ill and bedridden, or may be attributed as being part of the illness. Considering that the semicircular canals are more susceptible to vestibulotoxicity than the otoliths, head impulse testing and dynamic visual acuity could be used to identify and monitor bilateral vestibular function. ${ }^{[11]}$

Patients receiving ototoxic treatment should be monitored at regular intervals, which are medicine specific. Prevention of aminoglycoside-induced ototoxicity requires effective therapeutic drug monitoring, as well as hearing evaluation before, during and after drug treatment. Patients receiving platinum therapy should be evaluated prior to every cycle of chemotherapy. Additionally, monitoring should continue after treatment for at least 6 months (12 months for children $\leq 5$ years old) to detect late-onset ototoxicity dysfunction. ${ }^{[15]}$

Ototoxicity monitoring is premised on the fact that if a patient's auditory and/or vestibular symptoms are detected early, the clinician has an opportunity to explore alternatives to treatment to prevent ototoxicity. These options may include replacing an ototoxic drug with a safer, less ototoxic one (where feasible) or changing the drug administration schedule (e.g. from daily dosage to 3 times per week) to reduce the concentration in the inner ear. ${ }^{[2]}$

The success of ototoxicity monitoring depends on effective communication between all health professionals involved in the management of patients who are being treated with ototoxic medicines (the clinical pharmacist, audiologist and doctor). Patients benefit the most if the information from these professionals regarding ototoxicity monitoring is communicated coherently and consistently. It is a duty of care for both the audiologist and the physician to ensure that auditory-vestibular information is communicated in a manner (or format) that enables the doctor to make a clinical decision to prevent further ototoxic damage.

\section{New developments in the prevention of ototoxicity}

Some of the symptoms resulting from treatment with ototoxic medicines are irreversible and there is a need to protect patients from such effects. It is not always possible to prevent ototoxicity, even when patients are closely monitored during treatment. Because of this, alternative strategies are currently being developed and explored to work towards a future free of ototoxicity ${ }^{[16]}$ An obvious strategy is to discontinue the use of ototoxic medicines, thus eliminating the need for ototoxicity monitoring. However, some of the ototoxic medicines (e.g. cisplatin) are very effective therapeutic agents and therefore likely to remain in use until non-ototoxic alternatives become available.

Alternative strategies are needed to eliminate or reduce ototoxicity. Three approaches currently receiving attention from researchers are: (i) development of efficacious otoprotective pharmacological agents; (ii) reversing ototoxicity-induced symptoms using neurotrophins; and (iii) identification of improved genetic markers that can be used to reliably identify patients at higher risk of developing ototoxicity. Pharmacological agents such as D-methionine have been shown to exhibit otoprotective benefits and therefore hold much potential. ${ }^{[17]}$ Expression of certain neurotrophins in cochlear hair cells and neurotrophin receptors, selective tyrosine kinase receptor B (TrkB) and C (TrkC) and the low-affinity p75 receptor (a member of the tumour necrosis factor superfamily of receptors) in the spiral ganglion neurons, has been revealed. ${ }^{[18]}$

Otoprotective agents can be aimed at protecting patients with normal auditory-vestibular function at the start of treatment, while neurotrophins can be an intervention that targets restoration. ${ }^{[16]}$ Finally, identification of certain genetic markers via genetic screening 


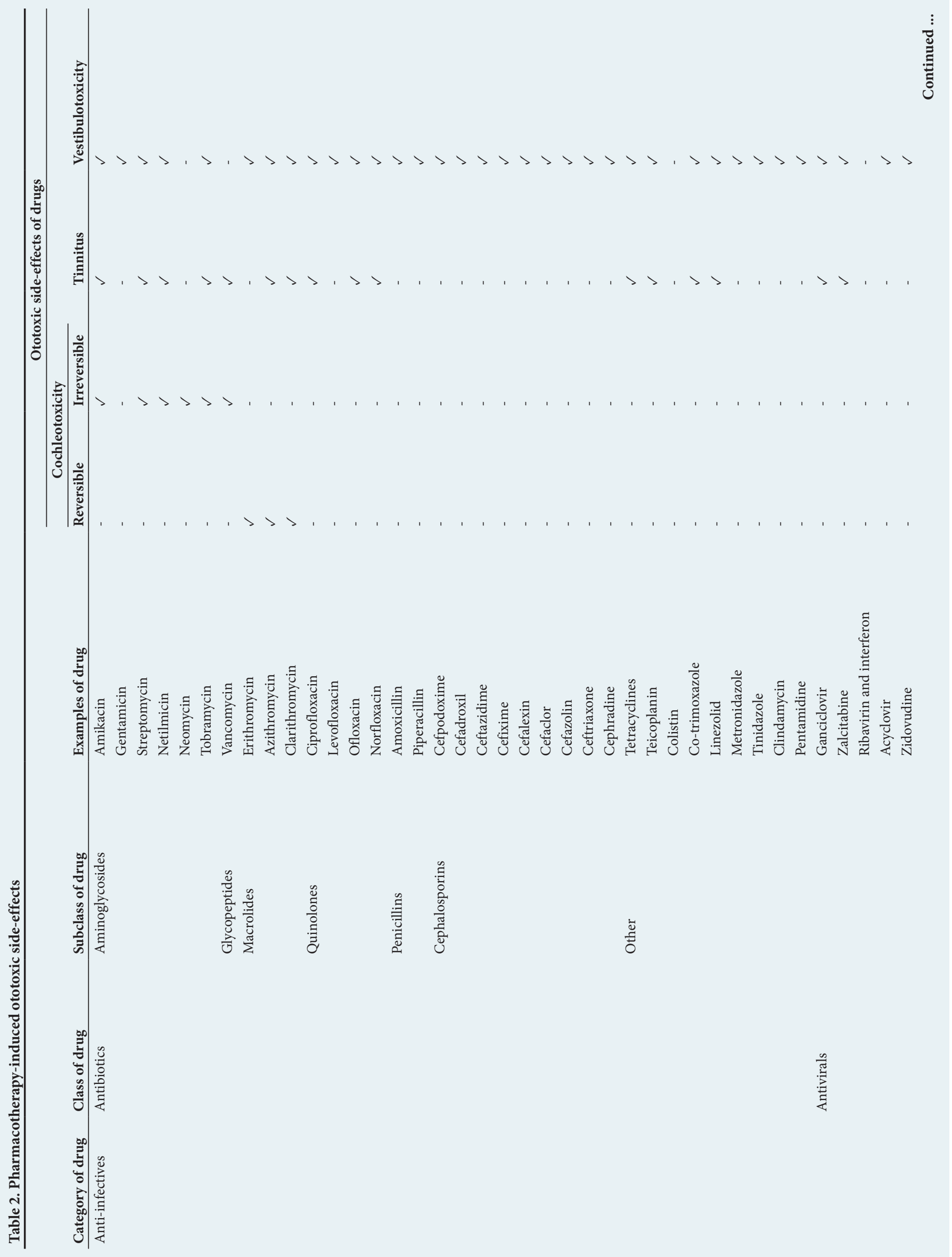




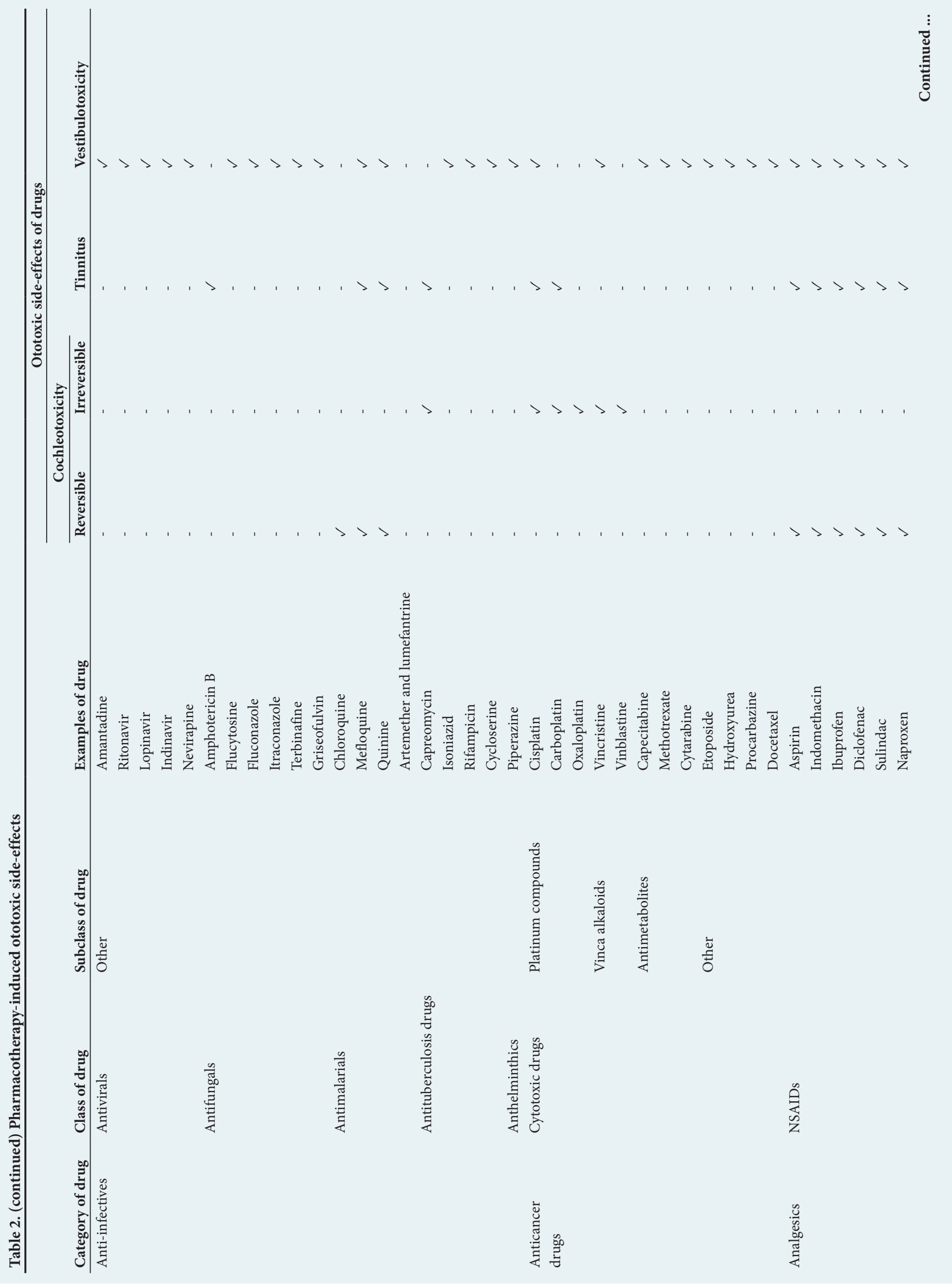




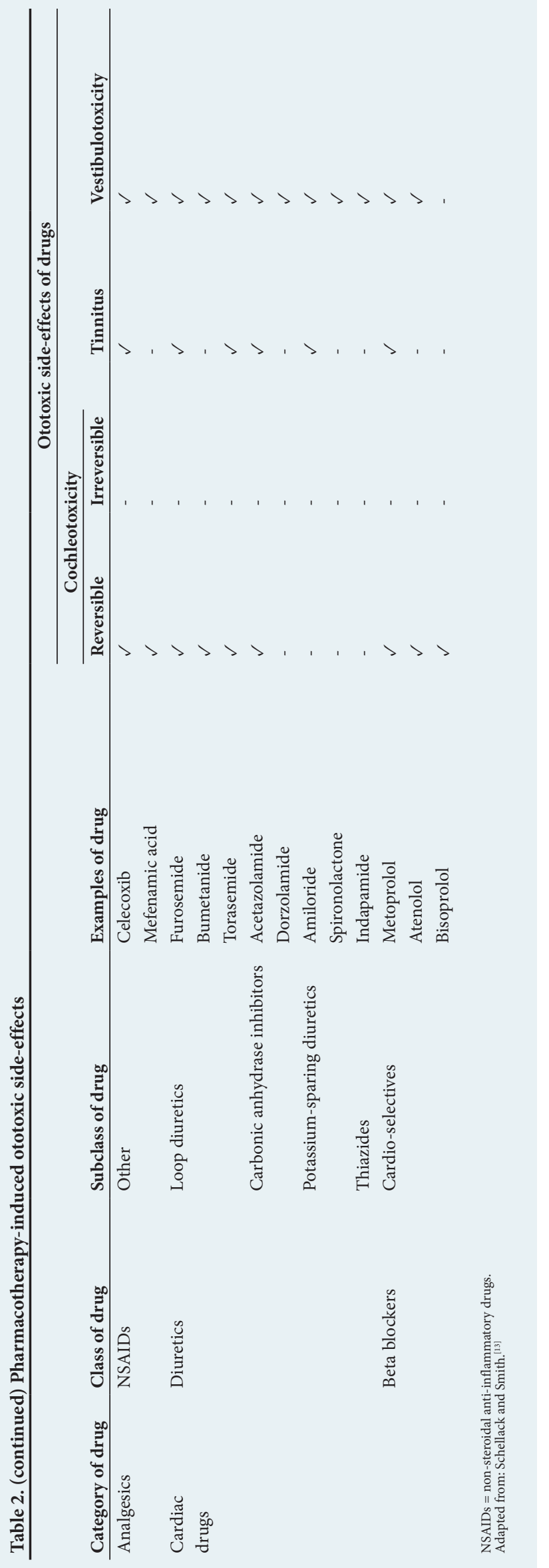

may facilitate easier identification of patients who are at higher risk of ototoxicity and for whom treatment with ototoxic medication is contraindicated. ${ }^{[19]}$

\section{Conclusion}

Ototoxicity is a serious and common complication of treating life-threatening illnesses such as cancer and multidrug-resistant tuberculosis. Currently, prospective monitoring of patients' hearing and vestibular function during treatment and instituting appropriate medical intervention when ototoxicity is first detected are the most effective strategies to prevent the debilitating effects of treatment. There are several areas of therapeutic development that hold enormous potential for eliminating the prospect of ototoxicity as an adverse outcome of treatment. Clinicians need to stay up to date with these developments.

\section{Declaration. None.}

Acknowledgements. None.

Author contributions. LR: conceptualised the project and wrote the first draft of the manuscript; NS: revised the manuscript and added pharmacyspecific content; $\mathrm{BH}$ : revised the manuscript and added vestibular toxicity content.

Funding. None.

Conflicts of interest. None.

1. Hacker M. Adverse drug reaction. In: Hacker M, Bachmann KA, Messer WS, eds. Pharmacology. Principles \& Practice. San Diego: Elsevier Science, 2009:327-351.

2. Bisht M, Bist SS. Ototoxicity: The hidden menace. Indian J Otolaryngol Head Neck Surg 2011;63(3):255-259. https://doi.org/10.1007/s12070-011-0151

3. Crundwell G, Gomersall P, Baguley DM. Ototoxicity (cochleotoxicity) classifications: A review. Int J Audiol 2016;55(2):65-74. https://doi.org/10.3109/14992027.2015.1094188

4. De Jager P, van Altena R. Hearing loss and nephrotoxicity in long-term aminoglycoside treatment in patients with tuberculosis. Int J Tuberc Lung Dis 2002;6(7):622-627.

5. Fausti SA, Henry JA, Helt WJ, et al. An individualized, sensitive frequency range for early detection 5. Fausti sA, Henry
of ototoxicity. Ear Hear 1999;20(6):497-505. https://doi.org/10.1097/00003446-199912000-00005

6. Styger PS, Hongzhe L. Systemic aminoglycosides are trafficked via endolymph into cochlear hair cells. Sci Rep 2011;1(1):159. https://doi.org/10.1038/srep00159

7. Knight KR, Kraemer DF, Winter C, et al. Early changes in auditory function as a result of platinum chemotherapy: Use of extended high frequency audiometry and evoked distortion product otoacoustic emission. J Clin Oncol 2007:25(10):1190-1195. https://doi.org/10.1200/jco.2006. 07.9723

8. Huth ME, Ricci, AJ, Cheng AG. Mechanisms of aminoglycosides ototoxicity and targets of hair cell protection. Int J Otolaryngol 2011;2011:1-19. https://doi.org/10.1155/2011/937861

9. Zingler VC, Weintz E, Jahn K, et al. Causative factors, epidemiology and follow-up of bilateral vestibulopathy. Ann NY Acad Sci 2009;1164(1):505-508. https://doi.org/10.1111/j.1749. 6632.2009.03765.x

10. Lin FR, Yaffe $K$, Jin Xia MS, et al. Hearing loss and cognitive decline in older adults. JAMA Intern Med 2013;173(4):293. https://doi.org/10.1001/jamainternmed.2013.1868

1. Smith PF, Darlington CL. Personality changes in patients with vestibular dysfunction. Front Hum Neurosci 2013;7:678. https://doi.org/10.3389/fnhum.2013.00678

12. Crumling MA, King KA, Duncan RK. Cyclodextrins and iatrogenic hearing loss: New drugs with significant risk. Front Cell Neurosci 2017;11:355. https://doi.org/10.3389/fncel.2017.00355

13. Schellack N, Smith N, Professional Board for Speech, Language and Hearing Professions. Management of patients on treatment that includes ototoxic medications: Guidelines 2018. Pretoria: Health Professions Council of South Africa, 2018.

14. Blakley BW, Cohen JI, Doolittle ND, et al. Strategies for prevention of toxicity caused by platinumbased chemotherapy: Review and summary of the annual meeting of the Blood-Brain Barrie Disruption Program, Gleneden Beach, Oregon, March 10, 2001. Laryngoscope 2002;112(11):19972001. https://doi.org/10.1097/00005537-200211000-00016

15. Konrad-Martin D, Gordon JS, Reavis KM, et al. Audiological monitoring of patients receiving ototoxic drugs. Perspect Hear Hear Disord Res Diagn 2005;9(1):17-22. https://doi.org/10.1044/ hhd9.1.17

16. Steyger PS, Cunningham LL, Esquivel CR, et al. Cellular mechanisms of ototoxicity. Front Cell Neurosci 2018;12:75. https://doi.org/10.3389/fncel.2018.00075

17. Campbell KC, Martin SM, Meech RP, et al. D-methionine (D-met) significantly reduces kanamycin induced ototoxicity in pigmented guinea pigs. Int J Audiol 2016;55(5):273-278. https://doi.org/10 3109/14992027.2016.1143980

18. Gao W-Q. Therapeutic potential of neurotrophins for treatment of hearing loss. Molec Neurol 1998;17(1-3):17-31. https://doi.org/10.1007/bf02802022

19. Ficher-Ghodsian N. Genetic factors in aminoglycoside toxicity. Ann NY Acad Sci 2005;884(1):99-1195. https://doi.org/10.1111/j.1749-6632.1999.tb08639.x 